\title{
Value of dual-source computed tomography in evaluating left ventricular function in patients with coronary heart disease
}

\author{
B.X. Shen ${ }^{1}$, D.H. Peng ${ }^{1}$, Y.G. Li ${ }^{1}$, Y. Liu' ${ }^{2}$ and Y. Gao ${ }^{2}$ \\ ${ }^{1}$ Department of Radiology, \\ Affiliated Nanshan Hospital of Guangdong Medical College, \\ Shenzhen, Guangdong, China \\ ${ }^{2}$ Ultrasound room, Affiliated Nanshan Hospital of Guangdong Medical College, \\ Shenzhen, Guangdong, China \\ Corresponding author: B.X. Shen \\ E-mail: bixianshen123@163.com
}

Genet. Mol. Res. 13 (2): 2417-2425 (2014)

Received February 5, 2013

Accepted June 26, 2013

Published April 3, 2014

DOI http://dx.doi.org/10.4238/2014.April.3.14

\begin{abstract}
We aimed to assess parameters reflecting left ventricular function by dual-source computed tomography (DSCT) with echocardiography (ECG) as control. Fifty-eight patients with coronary heart disease (CHD) were recruited from January to June 2011; 29 CHD patients had type II diabetes. All patients were assessed by cardiac DSCT and ECG examination. DSCT and ECG correlated well for ejection fraction $(\mathrm{EF})(\mathrm{r}=0.70)$, end-systolic volume (ESV) $(\mathrm{r}=0.87)$, stroke volume (SV) $(\mathrm{r}=0.83)$, and end-diastolic volume $(\mathrm{EDV})(\mathrm{r}=0.90)$. The mean ESV and EDV values measured by the two methods in CHD patients with type II diabetes were higher than those in non-diabetic patients, whereas the mean EF was lower. DSCT is an accurate and practical method for assessing left ventricular function.
\end{abstract}

Key words: Dual-source computed tomography; Echocardiography; Left ventricular function 


\section{INTRODUCTION}

Coronary heart disease (CHD), a common cardiovascular disease, is a serious human health issue that is a major cause of death worldwide. In the United States and Europe, CHD is the leading cause of left ventricular dysfunction and heart failure. In addition, left ventricular dilatation and global dysfunction in patients without symptoms of heart failure has been directly linked to the eventual development of symptomatic congestive heart failure in clinical and epidemiologic studies. Therefore, it is essential to assess left ventricular function accurately and reliably for CHD diagnosis, risk stratification, prognosis, and treatment (The Multicenter Postinfarction Research Group, 1983; White et al., 1987; Emond et al., 1994; Brener et al., 2006).

A variety of test methods, including echocardiography, single photon emission computed tomography (SPECT), perfusion, left ventricular angiography, and MR imaging may be used to assess left ventricular function in CHD patients. Among them, echocardiography is still the most widely used method in the clinic because it is convenient, rapid, economical, and does not use radiation, However, it is limited by the patients' ventricular shape, the technical ability of the operators, and the difficulty in dependably evaluating patients' coronary arteries. MRI has good spatial resolution and tissue contrast, does not use radiation, and can image in any direction. Some scholars consider MRI the gold standard for evaluating left ventricular function, but its clinical application is limited because the test procedure takes too long. In addition, most of today's MRI equipment cannot display the coronary artery very well. Unisource multilayer CT requires large doses of radiation and it is seldom used simply to evaluate heart function. If it is used to evaluate the coronary artery, 64 or more layers of CT are essential and, because of the impact of temporal resolution, the patient's heart rate has to be maintained at under 65 beats $/ \mathrm{min}$.

Currently, however, the temporal resolution of dual-source CT (DSCT) has reached $83 \mathrm{~ms}$, so heart rate is no longer an important factor affecting image quality. A one-time heart scan can not only display cardiac structure, but also allow assessment of cardiac function. In addition, the extent of coronary plaque and stenosis can be observed without additional contrast agents or radiation, and DSCT really becomes a one-stop screening method for the heart. There have been no reports comparing DSCT and ultrasound for the assessment of left ventricular function in CHD patients. We therefore used echocardiography as a control in our evaluations of DSCT for measuring left ventricular function in CHD patients.

\section{MATERIAL AND METHODS}

\section{Study objects}

Fifty-eight CHD patients in this hospital were selected from January to June in 2011. Among them, 29 patients had type II diabetes, 18 males and 11 females, aged 40 to 82 years old, with a mean age of $63 \pm 10$ years. Their diabetic histories ranged from 1 month to 20 years. The diagnostic criteria for diabetes were based on WHO 1999 criteria for type II diabetes. There were 29 non-diabetic CHD patients, 17 males and 12 females, aged 40 to 83 years, with a mean age of $67 \pm 11$ years. Among the CHD patients, there was no statistically significant difference in ages between the group with type II diabetes and the one without it. 
The patients in both groups had no history of alcohol use or smoking. All patients were examined by cardiac dual-source CT and echocardiography over three days. During this period, the patients' conditions were stable, and their treatment programs were unchanged. Cases with serious arrhythmia and respiratory artifacts were rejected.

\section{DSCT examination}

\section{CT scanning}

A Somatom Definition instrument (Siemens, Germany) was used for DSCT scanning. Procedures were explained and instructions given to the patients before examination; no drugs were used to control heart rate. The patients performed breathing exercises in a supine position after the ECG was connected to allow positioning imaging of the chest. A retrospective electrocardiogram gating technique was adopted as the coronary artery scanning program. The scanning range was from $1 \mathrm{~cm}$ below the tracheal bifurcation to the diaphragmatic surface of the heart. Calcification was scored with plain and enhanced scans. A binocular high-pressure syringe was used to inject $70-80 \mathrm{~mL}$ of non-ionic contrast agent (iopamidol; $370 \mathrm{mg} / \mathrm{mL}$ ) through the antecubital vein at $4-5 \mathrm{~mL} / \mathrm{s}$. The dose was adjusted according to the body weight of the patient and was followed by injection of $40 \mathrm{ml}$ saline at the same speed. Contrast agent tracking was performed, with the aortic root selected as the monitoring level. When the CT value of the region of interest was greater than $100 \mathrm{HU}$, the automatic scan was triggered after a 5-6 s delay. Scanning parameters: tube voltage, $120 \mathrm{kV}$; tube current, 380-400 mAs. The width of the collimating apparatus was $32 \times 0.6 \mathrm{~mm}$. Z-axis flying focus technology was used to collect 64 layers of $0.6 \mathrm{~mm} /$ layer. The matrix was $512 \mathrm{x}$ 512 , vision was $150 \times 150 \mathrm{~mm}-180 \times 180 \mathrm{~mm}$, the frame rotation time was $330 \mathrm{~ms}$, and the pitch was 0.2 to 0.5 . The parameters were automatically adjusted to accommodate changes in heart rate while scanning was in progress.

\section{Postprocessing and analysis of cardiac function}

After scanning, the original data were reconstructed on a deputy platform. Zero to $100 \%$ phase was reconstructed. With the interval at $10 \%$, we obtained images of 10 cardiac cycles. After completing the reconstruction, the image was transferred to the post-processing workstation. Circulation software was used for analysis, and the short axis of 10 cardiac cycles observed by film to determine end-diastole and end-systole. The intimal and outer membrane surfaces of the left ventricular cavity and left ventricular papillary muscle, including the blood pool, were outlined by automatic tuning and manually. Cardiac function analysis software was used to automatically calculate end-diastolic volume (EDV), end-systolic volume (ESV), stroke volume (SV), cardiac output (CO), ejection fraction (EF), and myocardial mass (MM). Analyses of the patients' cardiac function and coronary artery were completed by two experienced physicians.

\section{Reconstruction and analysis of coronary artery}

The highest quality images of the coronary artery were selected and circulation soft- 
ware applied to reconstruct coronary artery images of volume rendering (VR), multiple planar reformation (MPR), and maximum intensity projection (MIP). Fractionation method 15, as recommended by the American Heart Association, was used to analyze the coronary artery tree (Austen et al., 1975), the right coronary artery (RCA), including the 1st to 4th segments, the left main (LM) and left anterior descending arteries (LAD), including the 5th to 10th segments, and the circumflex artery (LCX), including the 11th to 15 th segments. The vessel diameter method was used to analyze arterial stenosis. Five stages were identified according to the degree of stenosis: stage 0 was no stenosis; stage 1 was $<50 \%$ stenosis (mild stenosis); stage 2 was $50-74 \%$ stenosis (moderate stenosis); stage 3 was $75-99 \%$ stenosis (severe stenosis); stage 4 was $100 \%$ for full occlusion. Each patient was graded according to their most severe instance of coronary stenosis.

\section{Echocardiography}

A PHILIPS CX50 Color ultrasound echocardiograph with a S5-1 probe was used on patients at rest to obtain views of the short axis of the aorta and parasternal long axis, as well as short axis views on each level, and of the standard apical four chambers, and section images of two chambers. Various indicators affecting left ventricular function were measured in the left parasternal chamber long axis. All the echocardiography of patients was performed by two experienced physicians.

\section{Statistical methods}

The SPSS17.0 statistical software was used to analyze the data. Quantitative data were expressed as mean \pm standard deviation. Paired-sample t-tests and correlation analyses were used to analyze the various indicators affecting left ventricular function of CHD patients, as measured by DSCT and echocardiography. The $t$-tests on two independent samples were used to analyze the corresponding data on CHD patients with and without type II diabetes. P $<0.05$ was considered statistically significant.

\section{RESULTS}

\section{Representation of left ventricular function in CHD patients examined by DSCT and echocardiography}

As shown in Figure 1, left ventricular function of a CHD patient (male, 72 years old) was examined by DSCT and echocardiography.

\section{Comparison of the indexes of left ventricular function in patients examined by DSCT and echocardiography}

The data show that the indexes for left ventricular function, including EF, ESV, EDV, and $\mathrm{SV}$, were well correlated in the two examination groups $(\mathrm{r}=0.70-0.90)$. There were no statistically significant differences in EF, ESV, EDV, or SV values between patients examined by echocardiography and those examined by DSCT $(\mathrm{P}>0.05)$ (Table 1$)$. 

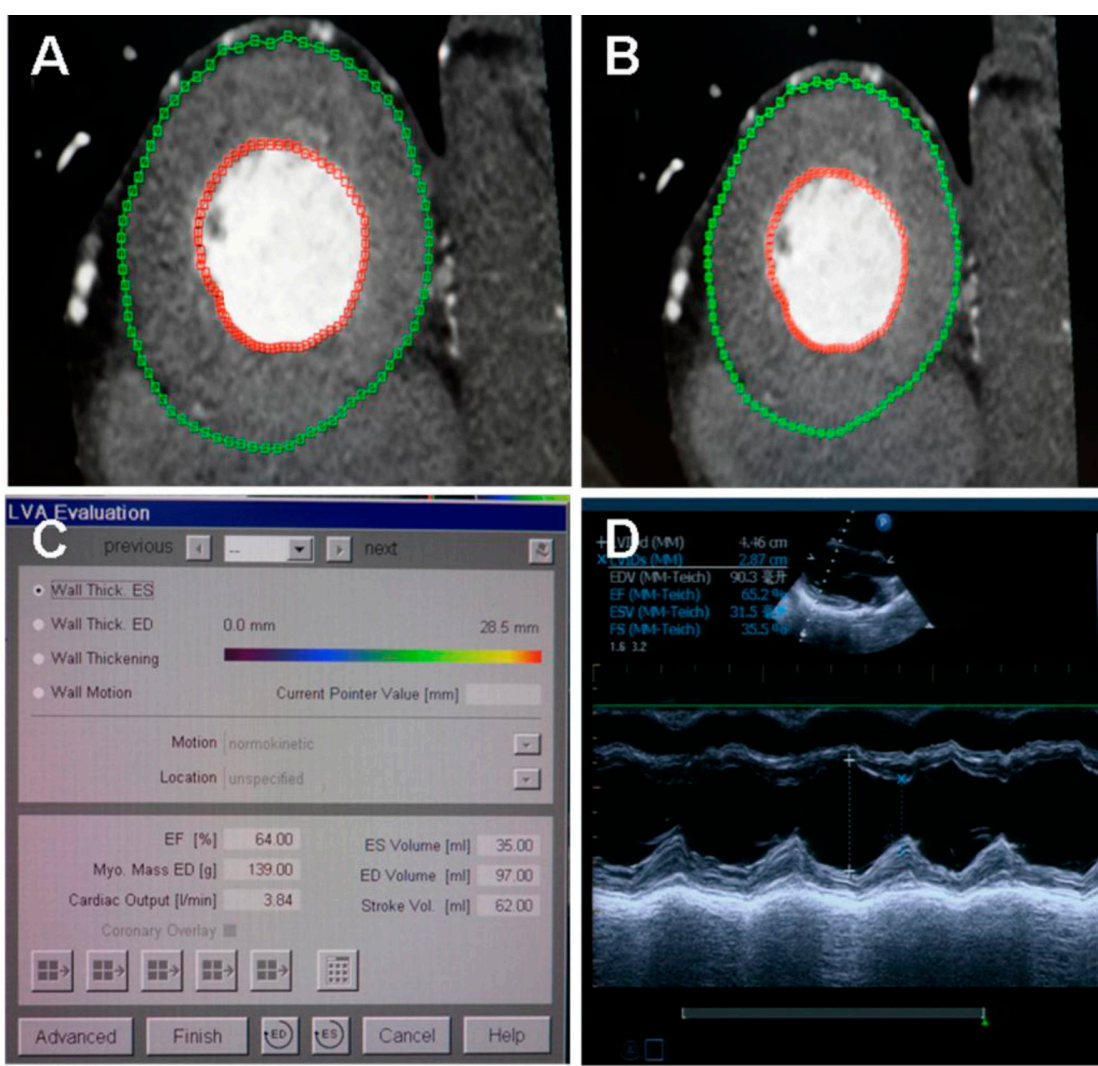

Figure 1. Male patient, 72 years old. A. Dual-source CT image of left ventricular short axis of the patient on enddiastolic stage. B. Dual-source CT image of left ventricular short axis of the patient on end-systolic stage. C. Dualsource CT measurement results of left ventricular function of the patient. D. Left ventricular function image and its results of this patient determined by M ultrasound measurement.

Table 1. Analysis results from the indexes reflecting left heart function of 58 patients measured by Dual-source CT and ECG.

\begin{tabular}{lcccc}
\hline & DSCT & ECG & r & P \\
\hline EF $(\%)$ & $61.53 \pm 7.46$ & $60.86 \pm 7.59$ & 0.70 & 0.381 \\
ESV $(\mathrm{mL})$ & $50.45 \pm 27.04$ & $51.59 \pm 26.23$ & 0.87 & 0.521 \\
EDV $(\mathrm{mL})$ & $125.69 \pm 37.12$ & $127.05 \pm 38.11$ & 0.90 & 0.544 \\
SV $(\mathrm{mL})$ & $75.36 \pm 15.22$ & $75.84 \pm 16.90$ & 0.83 & 0.702 \\
\hline
\end{tabular}

\section{Comparison of the indexes of left ventricular function in CHD patients with and without type II diabetes examined by echocardiography}

The ESV and EDV values for CHD patients with type II diabetes examined by echocardiography were significantly increased compared with those of CHD patients without type II diabetes, while the EF values were reduced $(\mathrm{P}<0.05)$ (Table 2). 


\begin{tabular}{|c|c|c|c|c|}
\hline & CHD with diabetes & CHD without diabetes & $t$ & $\mathrm{P}$ \\
\hline $\mathrm{EF}(\%)$ & $58.72 \pm 8.07$ & $63.00 \pm 6.53$ & 2.219 & 0.031 \\
\hline $\operatorname{ESV}(\mathrm{mL})$ & $58.76 \pm 32.95$ & $44.41 \pm 14.44$ & 2.147 & 0.036 \\
\hline $\mathrm{EDV}(\mathrm{mL})$ & $136.93 \pm 45.66$ & $117.17 \pm 25.87$ & 2.027 & 0.047 \\
\hline
\end{tabular}

\section{Comparison of the indexes of left ventricular function in CHD patients with and without type II diabetes examined by DSCT}

The ESV, EDV, and MM values of CHD patients with type II diabetes examined by DSCT were significantly increased compared with those of CHD patients without type II diabetes, while the EF values were reduced $(\mathrm{P}<0.05)$ (Table 3$)$.

\begin{tabular}{|c|c|c|c|c|}
\hline & CHD with diabetes & CHD without diabetes & $t$ & $\mathrm{P}$ \\
\hline $\mathrm{EF}(\%)$ & $58.76 \pm 8.34$ & $64.31 \pm 5.27$ & 3.031 & 0.004 \\
\hline $\mathrm{ESV}(\mathrm{mL})$ & $60.03 \pm 34.28$ & $40.86 \pm 11.11$ & 2.865 & 0.007 \\
\hline $\operatorname{EDV}(\mathrm{mL})$ & $137.48 \pm 44.77$ & $113.90 \pm 22.64$ & 2.532 & 0.014 \\
\hline $\mathrm{MM}(\mathrm{g})$ & $160.34 \pm 50.68$ & $125.14 \pm 35.68$ & 3.059 & 0.003 \\
\hline
\end{tabular}

\section{Analysis of coronary artery stenosis in CHD patients with or without diabetes}

The coronary artery image quality of the 58 patients was excellent. Coronary artery stenosis in CHD patients with type II diabetes was more severe than that of CHD patients without type II diabetes: 19 of 29 patients with diabetes had severe stenosis, compared with only 13 of 29 in patients without diabetes (Table 4).

\begin{tabular}{|c|c|c|c|}
\hline Pathological changes & CHD combined with diabetes & Without diabetes & Total $(\mathrm{N})$ \\
\hline Low grade & 5 & 8 & 13 \\
\hline Middle grade & 5 & 8 & 13 \\
\hline Severe grade & 11 & 9 & 20 \\
\hline Full occlusion & 8 & 4 & 12 \\
\hline Total & 29 & 29 & 58 \\
\hline
\end{tabular}

\section{DISCUSSION}

There are many methods for evaluating left ventricular function in CHD patients. Echocardiography is the most widely method applied in the clinic because it is convenient, rapid, economical, and does not require radiation. However, there are many limitations to its use, including when the patient's left ventricles are irregularly shaped. Measurement error is increased when, for example, heart chambers are expanded by myocardial infarction, myocardial hypertrophy, ventricular aneurysm formation, and local motion abnormalities, etc. (Qin et al., 2000; Jenkins et al., 2004; Lang et al., 2005). In addition, its accuracy is affected by the acoustic window and the proficiency of the operators. Some scholars consider MRI as the gold 
standard for evaluation of left ventricular function (Rathi and Biederman, 2004), but tests are expensive, its spatial resolution rate is not high, and because of its lengthy duration, it is difficult for patients to tolerate. In addition, emergency patients and those with heart pacemakers or metallic foreign bodies cannot be routinely examined by MRI. Domestic and foreign scholars (Yamamuro et al., 2005; Busch et al., 2008) used the results of MRI measurement on cardiac function as a standard with which to comparemulti-slice spiral CT MSCT and echocardiography in the measurement of left ventricular function. They found that MSCT is more accurate and reliable than ultrasound measurements of left ventricular function, and that spatial resolution in CT images is superior to MRI, allowing the inner and outer membrane profile of heart, myocardium, and its accessory structure to be displayed more clearly, and facilitating tracing the inner and outer membrane profiles of the heart. Previous studies showed that single-source MSCT did not obtain clear systolic and diastolic images from patients with a fast heart rate because of its low time resolution. This limited its application in the assessment of left ventricular function. The introduction of DSCT represented a leap forward for CT, shortening its time resolution to $83 \mathrm{~ms}$, and allowing clear images of systolic and diastolic phases to be obtained from patients with a fast heart rate without needing to give $\beta 2$-receptor blockers, which have adverse effects on cardiac function. In addition, data obtained from coronary angiography can be used to assess calcification scores, coronary artery stenosis, and valvular morphology, as well are to detect myocardial ischemia and infarction, and so on, without needing additional contrast agents or radiation (Ropers et al., 2007; Annuar et al., 2008; Brodoefel et al., 2008).

The results show that indexes of left ventricular function, including EF, ESV, EDV, and SV, are well correlated in patients examined by DSCT or echocardiography, with no statistically significant differences. This is consistent with the results of previous domestic and foreign studies (Uusitupa et al., 1990; Henneman et al., 2006; Salm et al., 2006; Butler et al., 2007; Annuar et al., 2008; Stolzmann et al., 2008). Compared with electrocardiographic measurements, the ESV, EDV, and SV values for patients measured by DSCT were slightly lower, while those for EF were slightly higher. Possible reasons include: 1) In DSCT examinations, the patient's emotional stress and the contrast agent injection can raise heart rate, which may affect the indexes of left ventricular function. 2) Results from echocardiography can be affected by the acoustic window and the experience of the equipment operators. 3) The measuring principles of the two examination methods are different: echocardiography utilizes the area length method, while DSCT utilizes the Simpson method. 4) The spatial resolution of echocardiography is low, and positioning may not be sufficiently accurate. 5) ECG and DSCT tests were not performed on the same day for some patients, so their blood flow dynamics might have changed between the tests.

The results from the two examination methods show that the indexes for left ventricular function, including ESV, EDV, and MM values, were increased in the diabetes group compared with those of the patients without diabetes. The EF values of the diabetic patients were lower, and the differences were statistically significant. This is a further indication that the DSCT and echocardiography results are consistent. The left ventricular function indexes for a normal person at rest are ESV about $55 \mathrm{~mL}$, EDV about $125 \mathrm{~mL}$, SV about $60-80 \mathrm{~mL}$, EF about 50-70\%, and MM about 90-133 g. The results of this study show that the indexes for left ventricular function in the CHD patients with type II diabetes were significantly reduced compared with those of the CHD patients without type II diabetes. This suggests that diabetes can lead to impairment of left ventricular function. Uddin et al. (2005) found the following 
pathological changes in the hearts of patients with type II diabetes: 1) microvascular disease; endothelial cell proliferation, degeneration, basement membrane thickening; 2) cardiac hypertrophy; diffuse myocardial fibrosis and focal necrosis of myocardial cells; 3) myocardial interstitium and perivascular fibrosis, and deposition of amino acid mucin-positive material. These changes can lead to myocardial stiffness, decreased ventricular compliance, myocardial cell necrosis, loss of a large number of contractile factors and lead to reduced ventricular systolic function. In addition, diabetes is a metabolic disorder that reduces energy production, and can result in barriers to $\mathrm{Ca}^{2+}$ transport and excitation-contraction coupling It can also lead to systolic dysfunction and decreased myocardial relaxation and compliance, leading to diastolic and systolic dysfunction of the left ventricle. Therefore, it is important to improve heart function by controlling the multiple risk factors of diabetes.

Domestic and international study results show that the pathological changes in the coronary artery of CHD patients with type II diabetes were more widespread and serious than those of non-diabetic CHD patients. The abnormal glucose metabolism in diabetes clearly aggravates the symptoms of CHD (Uddin et al., 2005). In this study, the degree of coronary stenosis of CHD patients with diabetes was more severe, indicating that diabetes is an important factor in the development of atherosclerosis.

In summary, DSCT is an accurate quantitative method for evaluating left ventricular function in CHD patients. Scanning can evaluate not only cardiac function, but also the coronary artery, which makes it superior to echocardiography. The indexes of heart function in the CHD patients with type II diabetes were different from those in non-diabetic CHD patients, and coronary artery stenosis was more severe. These conclusions are based on a small number of samples and need to be confirmed by a larger study.

\section{Conflicts of interest}

The authors declare no conflict of interest.

\section{REFERENCES}

Annuar BR, Liew CK, Chin SP, Ong TK, et al. (2008). Assessment of global and regional left ventricular function using 64-slice multislice computed tomography and 2D echocardiography: a comparison with cardiac magnetic resonance. Eur. J. Radiol. 65: 112-119.

Austen WG, Edwards JE, Frye RL, Gensini GG, et al. (1975). A reporting system on patients evaluated for coronary artery disease. Report of the ad hoc committee for grading of coronary artery disease, council on cardiovascular surgery, American Heart Association. Circulation 51: 5-40.

Brener SJ, Lytle BW, Casserly IP, Ellis SG, et al. (2006). Predictors of revascularization method and long-term outcome of percutaneous coronary intervention or repeat coronary bypass surgery in patients with multivessel coronary disease and previous coronary bypass surgery. Eur. Heart J. 27: 413-418.

Brodoefel H, Burgstahler C, Tsiflikas I, Reimann A, et al. (2008). Dual-source CT: effect of heart rate, heart rate variability, and calcification on image quality and diagnostic accuracy. Radiology 247: 346-355.

Busch S, Johnson TR, Wintersperger BJ, Minaifar N, et al. (2008). Quantitative assessment of left ventricular function with dual-source CT in comparison to cardiac magnetic resonance imaging: initial findings. Eur. Radiol. 18: 570-575.

Butler J, Shapiro MD, Jassal DS, Neilan TG, et al. (2007). Comparison of multidetector computed tomography and twodimensional transthoracic echocardiography for left ventricular assessment in patients with heart failure. Am. J. Cardiol. 99: 247-249.

Emond M, Mock MB, Davis KB, Fisher LD, et al. (1994). Long-term survival of medically treated patients in the Coronary Artery Surgery Study (CASS) Registry. Circulation 90: 2645-2657.

Henneman MM, Schuijf JD, Jukema JW, Holman ER, et al. (2006). Assessment of global and regional left ventricular 
function and volumes with 64-slice MSCT: a comparison with 2D echocardiography. J. Nucl. Cardiol. 13: 480-487. Jenkins C, Bricknell K, Hanekom L and Marwick TH (2004). Reproducibility and accuracy of echocardiographic measurements of left ventricular parameters using real-time three-dimensional echocardiography. J. Am. Coll. Cardiol. 44: 878-886.

Lang RM, Bierig M, Devereux RB, Flachskampf FA, et al. (2005). Recommendations for chamber quantification: a report from the American Society of Echocardiography's Guidelines and Standards Committee and the Chamber Quantification Writing Group, developed in conjunction with the European Association of Echocardiography, a branch of the European Society of Cardiology. J. Am. Soc. Echocardiogr. 18: 1440-1463.

Qin JX, Jones M, Shiota T, Greenberg NL, et al. (2000). Validation of real-time three-dimensional echocardiography for quantifying left ventricular volumes in the presence of a left ventricular aneurysm: in vitro and in vivo studies. J. Am. Coll. Cardiol. 36: 900-907.

Rathi VK and Biederman RW (2004). Imaging of ventricular function by cardiovascular magnetic resonance. Curr. Cardiol. Rep. 6: 55-61.

Ropers U, Ropers D, Pflederer T, Anders K, et al. (2007). Influence of heart rate on the diagnostic accuracy of dual-source computed tomography coronary angiography. J. Am. Coll. Cardiol. 50: 2393-2398.

Salm LP, Schuijf JD, de Roos A, Lamb HJ, et al. (2006). Global and regional left ventricular function assessment with 16-detector row CT: comparison with echocardiography and cardiovascular magnetic resonance. Eur. $J$. Echocardiogr. 7: 308-314.

Stolzmann P, Scheffel H, Trindade PT, Plass AR, et al. (2008). Left ventricular and left atrial dimensions and volumes: comparison between dual-source CT and echocardiography. Invest. Radiol. 43: 284-289.

The Multicenter Postinfarction Research Group (1983). Risk stratification and survival after myocardial infarction. $N$. Engl. J. Med. 309: 331-336.

Uddin SN, Malik F, Bari MA, Siddiqui NI, et al. (2005). Angiographic severity and extent of coronary artery disease in patients with type 2 diabetes mellitus. Mymensingh Med. J. 14: 32-37.

Uusitupa MI, Mustonen JN and Airaksinen KE (1990). Diabetic heart muscle disease. Ann. Med. 22: 377-386.

White HD, Norris RM, Brown MA, Brandt PW, et al. (1987). Left ventricular end-systolic volume as the major determinant of survival after recovery from myocardial infarction. Circulation 76: 44-51.

Yamamuro M, Tadamura E, Kubo S, Toyoda H, et al. (2005). Cardiac functional analysis with multi-detector row CT and segmental reconstruction algorithm: comparison with echocardiography, SPECT, and MR imaging. Radiology 234: 381-390. 